\title{
On some methods of researching the structural, physical and mechanical parameters of granular media as applied to the modeling of rock dumps
}

\author{
Vyacheslav Gogolin ${ }^{1, *}$, Yury Lesin ${ }^{2}$, and Ivan Košč ${ }^{3}$ \\ ${ }^{1}$ T.F. Gorbachev Kuzbass State Technical University, Department of Mathematics, 650000 \\ Kemerovo, 28 Vesennya st., Russian Federation \\ ${ }^{2}$ T.F. Gorbachev Kuzbass State Technical University, Department of Surveying and Geology, 650000 \\ Kemerovo, 28 Vesennya st., Russian Federation \\ ${ }^{3}$ Academy of the Police Force in Bratislava, Department of European Integrated Management of \\ Borders, 83517 Bratislava, 1 Sklabinska st, Slovakia
}

\begin{abstract}
The physical properties of the artificial disjointed rock massifs (the filling massifs and arrays of collapsed rocks and dumps) formed during mining operations, are largely determined by the geometry of the structure of pore space and the mineral phase, i.e. particle size, shape and packing method. In this case, the conditions for the arrangement of the particles relative to each other (the distances between the centers of the particles, the orientation, the number of contacts, the angles between the segments connecting the particle centers, etc.) have a decisive influence on the macroscopic characteristics of the rock mass (specific surface, bulk density, load capacity, etc.) and the behavior of such media under the applied stresses. Thus, in order to manage the state of disjointed technogenic rock massifs of bedrock, the question of applying modern methods for modeling bulk media for determining these parameters is considered. The article provides an overview of methods for modeling granular media to determine their structural, physical and mechanical parameters.
\end{abstract}

\section{Introduction}

The phenomenological approach, in which a discrete bulk medium is replaced by some fictitious continuous medium - a continuum characterized by observable averaged properties, measurable, continuous and differentiable by spatial coordinates and time, is generally accepted to describe processes occurring in bulk media. This approach was widely developed in the works of some authors devoted to solving the problems of bulk media mechanics and their applications in mining [1-6].

At the same time, the substance composing a bulk medium is heterogeneous both in its individual phases, which are homogeneous in themselves, and in larger aggregates. If a microscopic property of an individual solid or liquid particle obeys well known physical

\footnotetext{
${ }^{*}$ Corresponding author: gva.pm@kuzstu.ru
} 
laws, then the general, or macroscopic, property of the bulk medium itself is described by means of averaging parameters. Macroscopic properties cannot always be obtained from microscopic ones by statistical averaging.

In this regard, to research the processes occurring in bulk solids and essentially determined by their geometrical structure, it is advisable to use a structural approach, which allows to obtain the distribution of structural parameters of the medium and proceed to averaging at a qualitatively higher level, and also allows to build mathematical models to some extent reflecting the specificity of bulk media as a set of unconsolidated particles.

The diversity of real porous bodies, granular media and bulk materials requires the implementation of a number of simplifications in modeling their structure. However, such simplifications cannot be arbitrary, the model must preserve the geometric features of the structure determining the processes in the bulk medium. The degree of simplification depends on the research tasks. The most adequate models of bulk solids as a stochastic medium should reflect the statistically probabilistic nature of the parameters (size, coordinates) constituting the elements of the system [7].

\section{Methods of modeling}

In the optimal case, a model of the structure of a particular bulk material should be sufficiently universal and applicable to describe various properties and processes and at the same time be as convenient and simple as possible.

The adequacy of the model to the object is largely determined by the methods of estimating the parameters of the model and the accuracy allowed by the process. Adequacy of model and object by one or several parameters does not exclude a possibility of significant difference of their other properties.

A number of physical properties of a set of particles of crushed materials are determined by their morphological characteristics: size, shape and structure. The study of the relationship of particle size distribution and shapes of particles of bulk materials with their mechanical strength, density, bulk weight, stability of their packages, filtration capacity, etc. is the subject of research by the authors of works [8]. Attempts to obtain quantitative characteristics of the real shape of particles of grinding products have led to the conclusion about the expediency of using the ratio of three orthogonal dimensions $l: b: h$, where $l$ is length, $b$ is width, $h$ is height of the particle [9-11].

If the particles are large enough and it is possible to measure their volume experimentally, the shape characteristic may be supplemented by the value of the volume filling factor (coefficient of fullness of shape), anisometric coefficient [12], or numerical characteristic [9]. These and other particle shape criteria [13] only to some extent reflect its actual shape.

Published experimental data on measuring of particles of crushing and grinding products of different nature and their generalization using the mentioned size ratios are mostly of private character $[9,14,15]$. The few results of modeling of discrete media by polyhedrons $[16,17]$ known from literature concern the study of properties of individual particles, which does not always allow applying the obtained dependences to estimate the structural parameters of their aggregate.

Analysis of theoretical and experimental studies on modeling of discrete media using a system of polyhedrons of regular and irregular shape shows that the detailed consideration of bulk solids in conditions of impossibility of determining the shape of particles during technological works means exceeding the accuracy allowed by the process.

Thus, it is reasonable to use only one particle size - average diameter and its distribution - granulometric characteristic as input data when building a bulk material model. 
Problems on dense packing of spheres (3-dimensional case) and bodies of more complex shape belonging to the field of discrete geometry have been studied for quite a long time. At present, a number of papers have been published on the problems of arrangement of bodies in Euclidean space [13-25]. The problem of determining the filling of a dense packing of spheres does not yet have an exhaustive solution. Of practical interest is the statement that a dense packing of spheres of arbitrary granulometric composition cannot have greater porosity than a dense packing of identical spheres [12].

A complex system of granular media particles, modeled by a system of rigid spheres, is topologically disordered. Analytical description of such systems is not yet possible.

One of the most acceptable methods for solving practical problems when studying masses of fractured rocks, soils, bulk and granular materials is mathematical modeling. When studying physical processes occurring in granular media and depending on the structural parameters of the medium (porosity, specific surface, coordination number, pore geometry), which in turn are determined by the structure of the "skeleton" or pore space, the construction of mathematical structural models is currently the only way to establish quantitative characteristics and dependencies.

A large number of loose material packing models composed of spheres or other bodies of regular geometric shape are known from literature. All these models can be divided into two classes - deterministic and probabilistic.

In deterministic methods of structure modeling different approaches to the solution of the problem are possible. Historically, the first were the models of regular packings of the same spheres - hexagonal, cubic, rhombohedron, octahedron and others. These names were given by the type of elementary cells formed by lines connecting the centers of neighboring spheres. Regular packings are well studied. The porosity and density in such models are functions of the number of spheres in the calculated cell [5].

The method of transition to a unit cell based on the assumption of equality of parameters of systems with ordered and chaotic structures, if these structures are adequate and the properties of their components and their concentrations are the same (the notion of adequacy should be specified for each type of heterogeneous structures) is quite common. An ordered structure has a long-range order, in which case it is possible to identify a unit cell whose geometrical and physical properties are equal to the corresponding properties of the whole system. In the case where ordered and chaotic systems are characterized by the same parameters, have the same properties of components and their concentrations, to determine the effective properties of the heterogeneous material there is no need to perform averaging over a volume that has the properties of a representative element, i.e. the minimum volume of the heterogeneous system whose physical and geometric properties correspond to the properties of the whole array. The extraction of an elementary cell does not necessarily require a transition to an ordered structure. In the process of building a structural model of an inhomogeneous material, the equality of the average geometric parameters in the model and the real array should be maintained.

The applicability of the considered deterministic models to real bulk materials is limited by the fact that they do not take into account or do not fully account such factors as continuous particle size distribution and non-uniform particle packing. Real arrays of fractured rocks, soils and bulk materials are characterized by high inhomogeneity of particle size distribution and chaotic arrangement of particles. Such materials and massifs are statistical media, and it is reasonable to study their processes with probabilistic models.

Probabilistic models, in turn, can be divided into physical models, model of simple fluids as a system of metal spheres, and statistical models.

The essence of statistical modeling of bulk material structure is to calculate the coordinates and sizes of particles in some representative volume. The tool for solving this problem is the method of statistical tests (Monte Carlo method). 
B. Wood, F. Jacobson, W. Hoover and others studied the density of random packing of balls and its dependence on the representative volume, as well as the radial distribution function by the Monte Carlo method. The works [2, 14-16] are devoted to the random placement of bodies in two- and three-dimensional space.

In the method of statistical tests an organic combination of mathematical and experimental research methods is carried out, especially useful when studying the phenomenon of boundary effect, when the size of samples at least in one of the measurements is commensurable with the size of the filling capacity.

Thus, the range of questions solved by the method of statistical modeling is wide enough. This allows us to consider it as a versatile and promising method, with the help of which it is possible to solve important scientific problems of both theoretical and applied nature.

Recently, deterministic models based on the discrete element method (DEM) have become widespread. This method makes it possible to describe each particle of a disperse medium in static equilibrium or dynamic motion, taking into account its interaction with surrounding particles. The packing of particles of different sizes can be both ordered and chaotic.

The Tkachenko-Witten model [14] is a system of disks or spheres in contact without friction, so the application of this model is limited to the study of dense colloidal suspensions and suspensions. The model was created to calculate the distribution of loads (stresses) in a discrete medium. Based on the algorithm they developed, the weight of a newly packed particle is decomposed into two forces (by two holding contacts). The redistribution of these forces on all underlying particles is then calculated.

The application of DEM is convenient for studying the displacement of particles in granular media under the action of external forces. In the works of K. Bagi and I. Boitar $[15,16]$ the loading of a sample consisting of 100 chaotically and densely packed individual particles of round shape with known friction coefficients at the contact points of the particles, along one of the axes (Fig. 1, a) was studied. It was found that during packing deformation the medium forms groups of particles located close to each other and having approximately the same direction of possible displacement vector (Fig. 1, b). Such behavior of particles is characteristic of uncoherent granular medium, and cannot be reflected by the mechanics of continuous media.
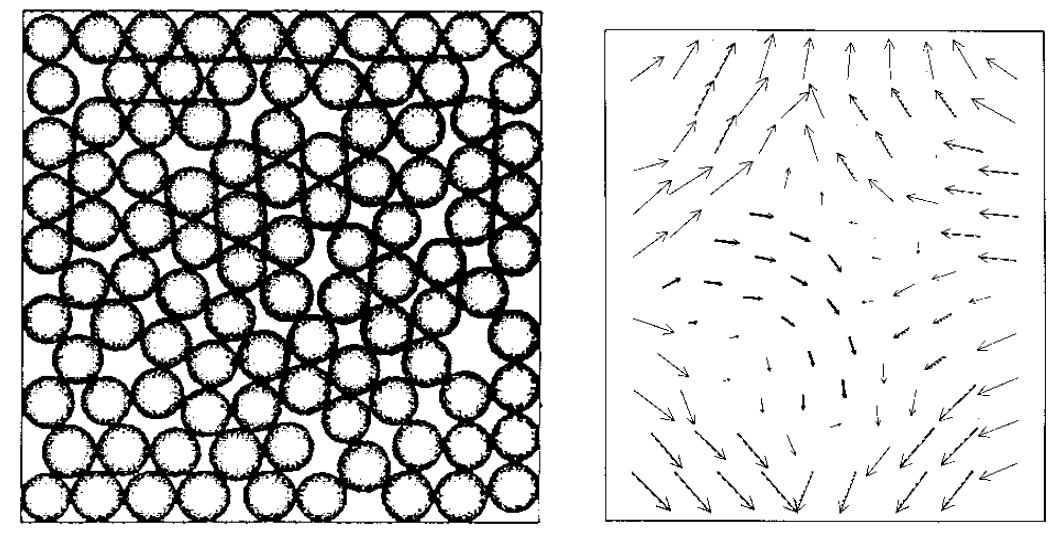

Fig. 1. Model of an incoherent granular medium (a) and vectors of possible movement of particles under the action of horizontally applied compressive load (b) 
Also, using DEM algorithms, it is possible to simulate the emergence and propagation of cracks in cohesive (cemented) rocks and the movement of emerging blocks relative to each other. For this purpose, the interaction parameters (cohesion and internal friction angle) between the particles are set, corresponding to the behavior of the medium as a continuous rather than granular one. The work [17] considers the process of fracture development in a fractured rock mass, leading to its destruction, and the mass is represented by a set of individual spherical particles. The interaction between the particles takes into account friction and adhesion forces, so their movement relative to each other is possible only when the Mohr-Coulomb equilibrium condition is violated, when shear forces begin to prevail over the forces holding the particles immobile relative to each other.

\section{Conclusion}

Practically all structural properties, such as radial distribution function and associated radial density function, porosity or filling density, specific surface area of the whole structure and its individual components, coordination characteristics of porous structures can be investigated using mathematical models of granular media. The coordination numbers can be calculated for the whole structure as well as for its individual components. This makes it possible to find the total number of links in the structure, to take into account their quality determined by the distance between the adjacent elements, to find the contact area between the array elements, to characterize the structure of pores formed by gaps between the elements of the modeled medium, the pore-size distribution, to determine the forces occurring at the contacts between particles and to determine the particle-to-particle motion in simulating medium fracture or deformation. Some of the named structural and mechanical characteristics are practically impossible to investigate by experimental methods.

Thus, the methods of statistical and discrete modeling of separate-grained media as well as of solid or fractured rock masses represent a powerful enough tool to research their strength properties. These methods can also be used to create modern composite materials with specified properties.

\section{References}

1. V.A. Gogolin, Yu.V. Lesin, Journal of Mining and Geotechnical Engineering, 3, 42 (2018)

2. I.A. Ermakova, Journal of Mining and Geotechnical Engineering, 1, 4 (2018)

3. T.D. Styles, Numerical Modeling and Analysis of slope stability within fracture dominated rock masses (Exeter, UK, University of Exeter, 2009)

4. R.D. Hart, An Introduction to distinct element modeling for rock engineering (in : Comprehensive Rock Engineering: Principles, Practice and Projects (Pergamon Press, London, 1993)

5. S. M. Rubio-Largo, P. G. Lind, D. Maza, R. C. Hidalgo, Comp. Part. Mech., 2, $127(2015)$

6. A. Tarokh, K. Chu-Shu, A. Fakhimi, J. F. Labuz, Comp. Part. Mech., 3, 391 (2016)

7. Y. T. Feng, D. R. J. Owen, Comp. Part. Mech., 1, 159 (2014)

8. W. L. Bragg, Journ. Sci. Instr., 19, 148, (1942)

9. M. R. J. Willie, G. H. F. Gardner, World Oil, 4, 121 (1958)

10. G. D. Bernal, G. Z. Finney, Nature, 214(5085), 265 (1967)

11. E. M. Tory, W. S. Jodrey, Adv. Mech. and Flow Granular Mater., 1, (1983) 
12. W. M. Visscher, M. Bolsteri, Nature, 239(5374) (1972)

13. P. L. Quang, D. L. Tien, T. P. Duc, T. N. Anh, Sustainable development of mountain territories, 11:4, 561 (2019)

14. A. V. Tkachenko, T. A. Witten, Phys. Rev. E, 60, 687 (1999)

15. K. Bagi, Acta Technica Acad. Sci. Hung., 107:1, 1 (1995)

16. K. Bagi, I. Bojtâr, Epiteses epiteszettudomany, 1-2, 75 (1989)

17. L. Scholtés, F.-V. Donzé, International Journal of Rock Mechanics \& Mining Sciences, 52, 18 (2012) 